Fisioter Bras 2020;21(6):565-70

https://doi.org/10.33233/fb.v21i6.4133

\title{
ARTIGO ORIGINAL \\ Avaliação da capacidade funcional, força muscular respiratória e pico de fluxo expiratório em pacientes com apneia obstrutiva do sono \\ Evaluation of functional capacity, respiratory muscular strength and expiratory flow peak in patients with obstructive sleep apnea
}

Kamila Giovanna da Conceição*, Marília Mendes Rodrigues ${ }^{\star \star}$, Kelly Christina de Faria Nunes, Ft., M.Sc. ${ }^{\star \star \star}$, Lays Magalhães Braga, Ft., M.Sc. ${ }^{* \star \star *}$

*Pós-graduanda em Fisioterapia Respiratória e Terapia Intensiva pelo Instituto Brasileiro de Reabilitação e Aprimoramento (IBRAESP), ${ }^{* *}$ Residente de Fisioterapia em Urgência e Emergência do Hospital Regional Antônio Dias (HRAD), Patos de Minas/MG, ${ }^{* * *}$ Doutoranda em Engenharia Biomédica pela UFU, Docente do Centro Universitário de Patos de Minas (UNIPAM), ****Doutoranda em Ciências pela Faculdade de Medicina da Universidade de São Paulo (FMUSP), Docente do Centro Universitário de Patos de Minas (UNIPAM)

Recebido em 16 de maio de 2020; aceito em 23 de novembro de 2020.

Correspondência: Kamila Giovanna da Conceição, Rua Eliezer José de Santana, 290 Cidade Nova 38706-402 Patos de Minas MG

Kamila Giovanna da Conceição: kmila_giovanna@hotmail.com

Marília Mendes Rodrigues: mariliamendesr@outlook.com

Kelly Christina de Faria Nunes: kellyfaria@unipam.edu.br

Lays Magalhães Braga: laysbraga@unipam.edu.br

\section{Resumo}

Introdução: A síndrome da apneia obstrutiva do sono (SAOS) é caracterizada pela obstrução das vias aéreas superiores durante o sono, o que causa efeitos sistêmicos durante a vigília. Objetivo: O objetivo deste estudo é avaliar a capacidade funcional, a força da musculatura respiratória e o pico de fluxo expiratório (PFE) de pacientes com SAOS. Métodos: Trata-se de um estudo transversal realizado na cidade de Patos de Minas/MG no ano de 2019, no qual foram incluídos 14 indivíduos que possuíam SAOS moderada e grave. Primeiramente os pacientes responderam a um questionário sociodemográfico elaborado pelos autores. Em seguida, foi realizada a manovacuometria, a mensuração do PFE e o teste de caminhada de seis minutos (TC6M). Resultados: A maioria dos pacientes eram homens $(71,4 \%)$, obesos $(78,6 \%)$ e hipertensos $(57,1 \%)$. Como resultados dos testes obtivemos - PImáx obtida vs predita $=86,4$ vs 102,8 ( $p=$ $0,004)$, PEmáx obtida vs predita $=98,5$ vs $113,5(p=0,05)$, PFE obtido vs predito $=381,3$ vs $495,8(p<0,001)$ e TC6M obtido vs predito $=485,3 \mathrm{~m}$ vs 536,6m $(p=0,03)$. Conclusão: Nessa amostra de indivíduos com SAOS grave e moderada houve redução da força muscular respiratória, do PFE, e da capacidade funcional.

Palavras-chave: síndromes da apneia do sono, músculos respiratórios, tolerância ao exercício.

Abstract

Introduction: Obstructive sleep apnea syndrome (OSAS) is characterized by upper airway obstruction during sleep, which causes systemic effects during wakefulness. Objective: The objective of this study is to assess functional capacity, respiratory muscle strength and peak expiratory flow (PEF) in patients with OSAS. Methods: This is a cross-sectional study held in the city of Patos de Minas/MG in the year of 2019, that included 14 individuals who had severe and moderate sleep apnea. First, the patients answered to a sociodemographic questionnaire prepared by the authors. Then, manovacuometry, PEF and six-minute walk test (6MWT) were performed. Results: Most patients were men (71.4\%), obese (78.6\%) and hypertensive $(57.1 \%)$. As results of the tests we obtained - MIP obtained vs predicted $=86.4$ vs $102.8(p=0.004)$, MPE obtained vs predicted $=98.5$ vs $113.5(p=0.05)$, PEF obtained vs predicted $=381.3$ vs 495.8 ( $p$ $<0.001)$ and 6MWT obtained vs predicted $=485.3 \mathrm{~m}$ vs $536.6 \mathrm{~m}(p=0.03)$. Conclusion: In this sample of individuals with severe and moderate OSAS, we observed a reduction in respiratory muscle strength, PEF, and functional capacity. 
Keywords: sleep apnea syndromes, respiratory muscles, exercise tolerance.

\section{Introdução}

A síndrome da apneia obstrutiva do sono (SAOS) é caracterizada pela obstrução parcial ou total das vias aéreas superiores (VAS) durante o sono. Tem como resultado a hipóxia, hipercapnia, e fragmentação do sono causando sintomas na vigília [1]. O ronco é o sinal mais evidente da síndrome ocorrendo no estágio mais profundo do sono quando o relaxamento muscular é maior [2]. A etiologia da SAOS é multifatorial, incluindo alterações anatômicas, craniofaciais e principalmente a obesidade que tem sido descrita como o maior fator de risco para oclusão da faringe e alteração da mecânica respiratória [3]. O diagnóstico da apneia do sono é realizado através do exame clínico e da polissonografia (PSG) que é considerada um exame padrão ouro. O tratamento usual para a SAOS é a aplicação da Pressão Positiva Contínua nas Vias Aéreas (CPAP) durante o sono. Esse aparelho possui várias interfaces que mantém as VAS abertas através da pressão positiva e é amplamente aceito como padrão para o tratamento, diminuindo ou eliminando assim as apneias. O principal problema é que o CPAP não tem uma boa adesão entre os pacientes mesmo com as novas tecnologias das máscaras [4].

A fraqueza dos músculos respiratórios pode causar dispneia e intolerância aos esforços, sendo assim de suma importância a avaliação dessa musculatura nos pacientes com SAOS, visto que as disfunções pulmonares podem estar presentes nesses indivíduos devido à má qualidade do sono. O colapso das vias aéreas superiores durante a noite acaba limitando o fluxo inspiratório e diminuindo o volume expiratório [5]. Os esforços repetitivos contra a obstrução das VAS e a hipóxia intermitente afetam negativamente os músculos inspiratórios dos pacientes com SAOS, e esses músculos se tornam significativamente mais fracos [6].

Alguns estudos randomizados [7-9] realizaram protocolos de treinamento da musculatura respiratória em pacientes com SAOS de diferentes graus, durante um período entre 5 e 12 semanas. Foi observado que o fortalecimento dessa musculatura contribuiu para a melhora da qualidade do sono e redução do número de apneias noturnas. Eles concluem que o treinamento é eficaz, simples, econômico e auxilia na redução da gravidade da síndrome. Por esse motivo é preciso realizar avaliações desses pacientes a fim de entender o comportamento da SAOS nos indivíduos e elaborar protocolos individuais de acordo com a necessidade de cada um.

Além dos prejuízos à musculatura respiratória, esses pacientes têm maior predisposição a eventos cardiovasculares adversos. As apneias e hipopneias são associadas ao estresse oxidativo criando uma cascata inflamatória aumentando os níveis de proteína $C$ reativa, a diminuição da atividade parassimpática e aumento da atividade simpática além de grandes oscilações de pressão intratorácicas. Essas oscilações negativas aumentam a pressão de todas as estruturas intratorácicas inclusive das câmaras cardíacas [10].

Tais consequências sugerem que a SAOS interfere de maneira sistêmica no organismo podendo afetar, inclusive, a capacidade funcional do paciente (aptidão que o indivíduo apresenta para a execução das atividades que garantem sua autonomia). Alguns estudos mostraram que a SAOS prejudica essa capacidade, enquanto outros sugeriram que ela continua preservada [11]. A capacidade funcional e consequentemente a tolerância ao exercício nos pacientes com SAOS ainda não é totalmente compreendida.

A medida do pico de fluxo expiratório (PFE) que mostra indiretamente resistência das VAS, ainda não é bem descrita na literatura na avalição de pacientes com apneia do sono. Somente um estudo [12] mensurou essa variável e encontrou similaridade entre os valores de pacientes com SAOS quando comparados a sujeitos saudáveis. Desta forma, faz-se necessário avaliar a capacidade funcional de exercício e a musculatura respiratória como um todo desses pacientes, a fim de compreender as suas limitações podendo assim nortear condutas de treinamento individualizadas para essa população, além de estratégias preventivas.

Assim, o presente estudo tem como objetivo avaliar a capacidade funcional, a força da musculatura respiratória e o PFE de pacientes com SAOS atendidos em uma clínica de Patos de Minas/MG.

Tratou-se de um estudo transversal de caráter quantitativo, aprovado pelo Comitê de Ética em Pesquisa do Centro Universitário de Patos de Minas - UNIPAM, sob parecer $\mathrm{n}^{\circ}$ 3.306.495. A amostragem foi do tipo não probabilística por conveniência. O período de recrutamento e coleta de dados foi realizado de junho a setembro de 2019 , à medida que 
contatamos os pacientes as avalições foram sendo realizadas de acordo com a disponibilidade dos mesmos. Foram incluídos no estudo voluntários que possuíam o diagnóstico clínico de SAOS de grau moderado a grave confirmado pelo exame de PSG, atendidos na Clínica do Sono localizada na cidade de Patos de Minas/MG, em uso ou não de CPAP, com idade $\geq 35$ e $\geq 70$ anos e concordância com termo de consentimento livre e esclarecido. $O$ estudo teve como critérios de exclusão: indivíduos que apresentaram patologias pulmonares, neuromusculares ou ortopédicas impeditivas à realização dos testes. Os pacientes foram orientados a comparecer em horário previamente agendado na Clínica Escola de Fisioterapia do UNIPAM onde foram avaliados e realizaram os testes propostos em um único momento.

Inicialmente foi aplicado um questionário geral elaborado pelos autores contendo informações sociodemográficas e clínicas como: idade, sexo, patologias prévias, além de questões relacionadas ao uso do CPAP. Em seguida o exame de PSG foi avaliado para a confirmação do diagnóstico de SAOS e a certificação do grau de apneia do sono.

Posteriormente, realizou-se a avaliação da força dos músculos respiratórios que foi mensurada pela manovacuometria, seguindo as recomendações metodológicas da American Thoracic Society/European Respiratory Society [13]. Para este teste foi utilizado o equipamento analógico da marca Wika ${ }^{\circledR}$ (Mv150). O paciente permaneceu sentado e com o clip nasal. Para a medida da pressão inspiratória máxima (PImáx) foi solicitada uma expiração a nível do volume residual seguida de um esforço inspiratório até cerca da capacidade pulmonar total (CPT). A pressão expiratória máxima (PEmáx) foi mensurada a partir da CPT, seguida de uma expiração máxima. Foram selecionadas três medidas que atendessem aos critérios de reprodutibilidade. A medida de maior valor foi considerada (com variação menor ou igual a $10 \%$ entre os demais). 0 cálculo do valor predito foi realizado em função da idade e sexo de acordo com Neder et al. [14].

Em seguida, a medida do PFE foi realizada por meio do aparelho Peak Flow (MiniWright ${ }^{\mathrm{TM}}$ ). O paciente foi posicionado sentado com clip nasal, em seguida solicitada uma inspiração em nível da CPT seguida de uma expiração rápida, na mesma velocidade da tosse [13]. As medidas foram coletadas três vezes, com intervalo de dois minutos entre elas e a de maior valor foi considerada. Os valores preditos foram de acordo com os estabelecidos por Leiner et al. [15].

Para finalizar, a capacidade funcional de exercício dos pacientes foi avaliada pelo teste de caminhada de seis minutos (TC6M). Para o início do teste foi dado um intervalo de repouso (10 a 15 minutos) para que o paciente se recuperasse e não houvesse interferência dos testes realizados anteriormente a esse. Os pacientes foram orientados a caminhar em um corredor demarcado de 30 metros, no maior ritmo tolerado. O TC6M foi realizado seguindo as recomendações da American Thoracic Society [16]. A distância predita para cada paciente foi calculada por meio da equação proposta por Enright e Sherrill [17].

A análise estatística foi conduzida por meio do Software Statistical Package for the Social Sciences 23.0. A normalidade dos dados foi testada por meio do teste Shapiro-Wilk. Para a análise descritiva dos dados foi empregada média \pm desvio padrão, números absolutos e frequências (\%). Para comparar os valores obtidos com os preditos foi empregado o Teste $t$ Student para amostras independentes e adotado um nível de significância de $5 \%(p<0,05)$.

Resultados

Foram recrutados para o estudo 20 indivíduos, destes, seis foram excluídos durante as avaliações por não atenderem aos critérios de inclusão, tais como apneia de grau leve (2), idade fora da estabelecida (3) e patologia ortopédica (1). Ao fim, a amostra foi composta por 14 voluntários.

Em relação à adesão ao uso do CPAP, 11 pacientes $(78,6 \%)$ relataram utilizar o recurso, e três $(21,4 \%)$ não faziam o uso do mesmo devido ao custo elevado e ao incômodo da máscara. Somente um indivíduo realizava acompanhamento com fisioterapeuta da área do sono, e o restante, 13 indivíduos (92,9\%), não possuíam nenhum tipo de acompanhamento.

A caracterização da amostra dada pelo questionário geral está apresentada na Tabela I. Dentre os pacientes, 11 possuíam SAOS grave sendo dois desses com sobrepeso e nove com obesidade. 
Tabela I - Características clínicas e sociodemográficas dos participantes com SAOS $(N=14)$. Patos de Minas; 2019.

\begin{tabular}{|c|c|}
\hline & Participantes $n=14$ \\
\hline Idade, anos & $55,7 \pm 12,0$ \\
\hline Sobrepeso & $3(21,4 \%)$ \\
\hline Obesidade & $11(78,6 \%)$ \\
\hline Sexo, n (\%) & \\
\hline Feminino & $4(28,6 \%)$ \\
\hline Masculino & $10(71,4 \%)$ \\
\hline Patologias associadas, $\mathbf{n}(\%)$ & \\
\hline Hipertensão & $8(57,1 \%)$ \\
\hline Diabetes & $4(28,6 \%)$ \\
\hline Depressão & $3(21,4 \%)$ \\
\hline Disfunções tireoidianas & $2(14,3 \%)$ \\
\hline Tabagismo & \\
\hline Sim & $2(14,3 \%)$ \\
\hline Não & $9(64,3 \%)$ \\
\hline Ex-tabagista & $3(21,4 \%)$ \\
\hline Realiza exercício físico, $n(\%)$ & \\
\hline $\mathrm{Sim}^{\star}$ & $6(42,9 \%)$ \\
\hline Não & $8(57,1 \%)$ \\
\hline G rau da SAOS, $n(\%)$ & \\
\hline Grave & $11(78,6 \%)$ \\
\hline Moderada & $3(21,4 \%)$ \\
\hline
\end{tabular}

$\mathrm{N}=$ número de pacientes; \%: porcentagem; \pm : desvio padrão; SAOS: síndrome da apneia obstrutiva do sono; *: mínimo de 3 vezes por semana. Fonte: Autores.

$\mathrm{Na}$ Tabela II, são apresentados os valores obtidos e preditos da manovacuometria, PFE e TC6M. Foi observada uma diferença estatisticamente significativa em todas as variáveis obtidas quando comparadas aos valores preditos.

Tabela II - Valores obtidos e preditos dos testes realizados no estudo. Indivíduos com SAOS (N = 14). Patos de Minas, 2019.

\begin{tabular}{llll}
\hline Variáveis & $\begin{array}{l}\text { Valores preditos } \\
\text { Média } \pm \mathrm{DP}\end{array}$ & $\begin{array}{l}\text { Valores obtid os } \\
\text { Média } \pm \mathrm{DP}\end{array}$ & Valor de $\mathbf{p}$ \\
\hline Plmáx & $102,8 \pm 16,9$ & $86,4 \pm 28,4$ & 0,004 \\
PEmáx & $113,5 \pm 28,4$ & $98,5 \pm 20,7$ & 0,05 \\
PFE & $495,8 \pm 75,4$ & $381,4 \pm 106,5$ & 0,001 \\
TC6M & $536,6 \pm 105,5$ & $485,3 \pm 65,9$ & 0,03 \\
\hline
\end{tabular}

PImáx = pressão inspiratória máxima; PEmáx = pressão expiratória máxima; PFE = pico de fluxo expiratório; TC6M = teste de caminhada de 6 minutos; $\mathrm{DP}=$ desvio padrão; $p=$ valor obtido pelo teste $\mathrm{t}$ independente, sendo estatisticamente significativa a diferença para $p<0,05$. Fonte: Autores.

\section{Discussão}

Alguns estudos apontam a maior prevalência de SAOS no sexo masculino e a obesidade como o maior fator de risco [18-20], corroborando os achados deste estudo.

Para Lim et al. [19] existem diferenças na distribuição de gordura corporal entre homens e mulheres. Em homens obesos, a deposição de gordura ocorre na parte superior do corpo, o que implica maior acúmulo de tecido adiposo na região do abdômen e pescoço. O estudo de Valipour [20] sugere que os hormônios femininos como a progesterona, parecem ter efeito protetor sobre a faringe, reduzindo o risco de colapso durante o sono.

A obesidade e a SAOS são fatores que potencialmente alteram a capacidade aeróbica e a tolerância ao exercício. Assim como em nosso estudo, na revisão sistemática de Mendelson et al. [21] ficou claro que a capacidade máxima ao exercício era prejudicada em pacientes com SAOS quando comparados com controles e quando expressos em relação aos valores preditivos. Já, o estudo de Carvalho et al. [3] verificou que a distância percorrida nos testes de shuttle e TC6M, foi inferior à distância de referência para indivíduos saudáveis na população brasileira, indicando que os pacientes obesos e com SAOS apresentavam uma redução na tolerância ao exercício. 
Além da redução na capacidade funcional, o presente estudo teve como achado a diminuição dos valores de força muscular respiratória e PFE. Resultados divergentes ao de Tassinari et al. [12], em que foram avaliados 19 indivíduos com SAOS e não foi observado prejuízo na função pulmonar e na força da musculatura respiratória. Entretanto, a população estudada por eles não incluía pacientes obesos e sim indivíduos com sobrepeso, diferentemente deste estudo no qual mais de $78 \%$ dos pacientes tinham obesidade. Acredita-se que a redução do PFE está relacionada à redução da capacidade funcional dos pacientes corroborando o achado de Carvalho et al. [3], que observaram uma diminuição do VEF1 avaliado pela espirometria, e associou esse achado com a menor tolerância ao esforço, comprovado também pelo TC6M.

Em relação às patologias apresentadas, a mais prevalente foi a hipertensão. Na pesquisa de Kanda et al. [22] foi analisado o perfil clínico, antropométrico e metabólico de pacientes com apneia do sono, na qual $90 \%$ da população estudada apresentava HAS, semelhante aos nossos achados. O estudo epidemiológico sobre a relação entre SAOS e HAS mais citado na literatura é o coorte de Peppard et al. [23] que acompanharam 709 indivíduos por oito anos e encontraram uma associação entre a SAOS e a hipertensão, mesmo em pacientes com apneia do sono leve e moderada. A maioria das evidências verifica a relação positiva entre SAOS e hipertensão, doença arterial coronariana, fibrilação atrial, acidente vascular cerebral e insuficiência cardíaca [24].

Este estudo possui limitações, entre elas estão o tamanho reduzido da amostra, escassez de estudos recentes sobre a função pulmonar de indivíduos com apneia do sono, avaliação de somente um grupo de pacientes com SAOS não havendo um grupo controle de obesos sem SAOS, e o uso do CPAP por alguns indivíduos. Desta forma, estudos com amostras maiores, ensaios clínicos randomizados e metodologia rígida são imprescindíveis para a elucidação da fisiopatologia da síndrome, principalmente no que se refere a comprometimentos pulmonares já que a literatura possui uma maior variedade de artigos no âmbito de comprometimento cardiovascular na SAOS.

Conclusão

Conclui-se que na amostra de indivíduos com apneia do sono grave e moderada, a força muscular respiratória, o pico de fluxo expiratório e a capacidade funcional mostraram-se reduzidas. Essas alterações podem indicar a necessidade de intervenções fisioterapêuticas para a redução de fatores de risco cardiopulmonares e estratégias de treinamento mais específicas para essa população.

Referências

1. Wimms A, Woehrle H, Ketheeswaran S, Ramanan D, Armitstead J. Obstructive sleep apnea in women: specific issues and interventions. Biomed Res Int 2016:1-9. https://doi.org/10.1155/2016/1764837

2. Silva MMM, Tavares TE, Pinto VSR. A relação entre a apneia e hipopneia obstrutiva do sono, respiração oral e obesidade com enfoque no tratamento fonoaudiológico: um estudo bibliográfico. Disturb Comun 2015;27(2):355-363. Disponível em: https://revistas.pucsp.br/index.php/dic/article/view/20790

3. Carvalho TMCS, Soares AF, Climaco DCS, Secundo IV, Lima AMJ. Associação entre função pulmonar, força muscular respiratória e capacidade funcional de exercício em indivíduos obesos com síndrome da apneia obstrutiva do sono. J Bras Pneumol 2018;44(4):279-84. https://doi.org/10.1590/s1806-37562017000000031

4. Zhang M, Liu Y, Liu Y, Yu F, Yan S, Chen L et al. Effectiveness of oral appliances versus continuous positive airway pressure in treatment of OSA patients: An updated meta-analysis. Cranio 2019;37(6):347-64. https://doi.org/10.1080/08869634.2018.1475278

5. Butner KL, Hargens TA, Kaleth AS, Miller L, Zedalis D, Herbert WG. Association of obstructive sleep apnea severity with exercise capacity and health-related quality of life. North Am J Med Sci 2013;5(6):362-6. https://doi.org/10.4103/1947-2714.114168

6. Kline CE, Crowley EP, Ewing GB, Burch JB, Blair SN, Durstine JL et al. The effect of exercise training on obstructive sleep apnea and sleep quality: a randomized controlled trial. Sleep 2011;34(12):1631-40. https://doi.org/10.5665/sleep.1422 
7. Lin HC, Chiang LL, Ong JH, Tsai K, Hung CH, Lin CY. The effects of threshold inspiratory muscle training in patients with obstructive sleep apnea: a randomized experimental study. Sleep Breathing 2019;24(1):201-9. https://doi.org/10.1007/s11325019-01862-y

8. Souza AKF, Andrade AD, Medeiros AIC, Aguiar MIR, Rocha TDS, Pedrosa RP et al. Effectiveness of inspiratory muscle training on sleep and functional capacity to exercise in obstructive sleep apnea: a randomized controlled trial. Sleep Breath 2017;22(3):63139. https://doi.org/10.1007/s11325-017-1591-5

9. Kuo YC, Song TT, Bernard JR, Liao YH. Short-term expiratory muscle strength training attenuates sleep apnea and improves sleep quality in patients with obstructive sleep apnea. Respir Physiol Neurobiol 2017;243:86-91. https://doi.org/10.1016/j.resp.2017.05.007

10. Javaheri S, Barbe F, Rodriguez FC, Dempsey JA, Khayat R, Javaheri S et al. Sleep apnea. Journal of the American College of Cardiology 2017;69(7):841-58. https://doi.org/10.1016/j.jacc.2016.11.069

11. Berger M, Kline CE, Cepeda FX, Rizzi CF, Chapelle C, Laporte S et al. Does obstructive sleep apnea affect exercise capacity and the hemodynamic response to exercise? An individual patient data and aggregate meta-analysis. Sleep Medicine. 2019;45:42-53. https://doi.org/10.1016/..smrv.2019.03.002

12. Tassinari CCR, Piccin CF, Beck MC, Scapini F, Oliveira LCA, Signor LU et al. Capacidade funcional e qualidade de vida entre sujeitos saudáveis e pacientes com apneia obstrutiva do sono. Rev USP Medicina 2015;49(2):152-9. https://doi.org/10.11606/issn.2176-7262.v49i2p152-159

13. American Thoracic Society/European Respiratory Society. ATS/ERS. Statement on respiratory muscle testing. Am J Respir Crit Care Med 2002;166(4):518-624. https://doi.org/10.1164/rccm.166.4.518

14. Neder JA, Andreoni S, Lerario MC, Nery LE. Reference values for lung function tests. II. Maximal respiratory pressures and voluntary ventilation. Braz J Med Biol Res 1999;32(6):719-27. https://doi.org/10.1590/S0100-879X1999000600007

15. Leiner GC, Abramowitz S, Small MJ, Stenby VB, Lewis WA. Expiratory peak flow rate. Standard values for normal subjects. Use as a clinical test of ventilatory function. Am Rev Respir Dis 1963:644-51. https://doi.org/10.1164/arrd.1963.88.5.644

16. American Thoracic Society. Guidelines for the six-minute walk test. Am J Respir Crit Care Med. Statement 2002;166(1):111-7. https://doi.org/10.1164/ajrccm.166.1.at1102

17. Enright PL, Sherrill DL. Reference equations for the six-minute walk in healthy adults. Am J Respir Crit Care Med 1998;158(5):1384-87. https://doi.org/10.1164/ajrccm.158.5.9710086

18. Hein M, Lanquart JP, Loas G, Hubain P, Linkowski P. Prevalence and risk factors of moderate to severe obstructive sleep apnea syndrome in insomnia sufferers: a study on 1311 subjects. Resp Res 2017;18(1):1-10. https://doi.org/10.1186/s12931-017-0616-8

19. Lim YH, Choi J, Kim KR, Shin J, Hwang KG, Ryu S et al. Sex-specific characteristics of anthropometry in patients with obstructive sleep apnea. Ann Otol Rhinol Laryngol 2014;123(7):517-23. https://doi.org/10.1177/0003489414526134

20. Valipour A. Gender-related differences in the obstructive sleep apnea syndrome. Pneumologie 2012;66(10):584-8. https://doi.org/10.1055/s-0032-1325664

21. Mendelson M, Marillier M, Bailly S, Flore P, Borel J-C, Vivodtzev I et al. Maximal exercise capacity in patients with obstructive sleep apnea syndrome: a systematic review and meta-analysis. Eur Resp J 2018;51(6):1-51. https://doi.org/10.1183/13993003.02697-2017

22. Kanda GM, Priore NC, Toledo C, Shimizu RN, Arata YP, Gonzaga C et al. Perfil clínico e laboratorial de pacientes com e sem apneia obstrutiva do sono. Rev Soc Bras Clin Med 2018;16(2):108-112. Disponível em: http://docs.bvsalud.org/biblioref/2018/09/913371/162108-112.pdf

23. Peppard PE, Young T, Palta M, Skatrud J. Prospective study of the association between sleep-disordered breathing and hypertension. New Eng $\mathrm{J}$ of Med 2000;342(19):1378-84. https://doi.org/10.1056/NEJM200005113421901

24. Gonzaga C, Bertolami A, Bertolami M, Amodeo C, Calhoun D. Obstructive sleep apnea, hypertension and cardiovascular diseases. J Hum Hypertens 2015;29(12):705-12. https://doi.org/10.1038/jhh.2015.15 